\title{
SPARSE RECONSTRUCTION BY SEPARABLE APPROXIMATION
}

\author{
Stephen J. Wright ${ }^{(a)}$, Robert D. Nowak ${ }^{(b)}$ \\ (a) Computer Sciences Department \\ (b) Dept. of Electrical and Computer Eng. \\ University of Wisconsin-Madison \\ Madison, WI, USA
}

\author{
Mário A. T. Figueiredo \\ Instituto de Telecomunicações \\ Dept. of Electrical and Computer Eng. \\ Instituto Superior Técnico \\ Lisboa, Portugal
}

\begin{abstract}
Finding sparse approximate solutions to large underdetermined linear systems of equations is a common problem in signal/image processing and statistics. Basis pursuit, the least absolute shrinkage and selection operator (LASSO), wavelet-based deconvolution and reconstruction, and compressed sensing (CS) are a few well-known areas in which problems of this type appear. One standard approach is to minimize an objective function that includes a quadratic $\left(\ell_{2}\right)$ error term added to a sparsity-inducing (usually $\ell_{1}$ ) regularizer. We present an algorithmic framework for the more general problem of minimizing the sum of a smooth convex function and a nonsmooth, possibly nonconvex, sparsity-inducing function. We propose iterative methods in which each step is an optimization subproblem involving a separable quadratic term (diagonal Hessian) plus the original sparsity-inducing term. Our approach is suitable for cases in which this subproblem can be solved much more rapidly than the original problem. In addition to solving the standard $\ell_{2}-\ell_{1}$ case, our approach handles other problems, e.g., $\ell_{p}$ regularizers with $p \neq 1$, or group-separable (GS) regularizers. Experiments with CS problems show that our approach provides state-of-the-art speed for the standard $\ell_{2}-\ell_{1}$ problem, and is also efficient on problems with GS regularizers.
\end{abstract}

Index Terms - sparse approximation, compressed sensing, optimization, reconstruction.

\section{INTRODUCTION}

\subsection{Problem Formulation}

There is growing interest in finding fast algorithms for solving the convex unconstrained optimization problem

$$
\min _{\mathbf{x} \in \mathbb{R}^{n}} \frac{1}{2}\|\mathbf{y}-\mathbf{A x}\|_{2}^{2}+\tau\|\mathbf{x}\|_{1},
$$

where $\mathbf{y} \in \mathbb{R}^{k}, \mathbf{A} \in \mathbb{R}^{k \times n}$ (usually $k<n$ ) and $\tau \in \mathbb{R}^{+}$. Problems of the form (1) can be used to identify a sparse approximate solution to the underdetermined system $\mathbf{y}=\mathbf{A x}$, and have become familiar over the past three decades, particularly in signal processing. Several algorithms have been proposed for solving (1) and its variants; see [15] for a recent overview of the work in this domain.

In this paper we propose algorithms for solving the following generalization of the problem (1):

$$
\min _{\mathbf{x}} \phi(\mathbf{x}):=f(\mathbf{x})+\tau c(\mathbf{x}),
$$

where $f: \mathbb{R}^{n} \rightarrow \mathbb{R}$ is a smooth and convex function, and $c: \mathbb{R}^{n} \rightarrow \mathbb{R}$, usually called the regularizer or regularization term, is finite for all $\mathbf{x} \in \mathbb{R}^{n}$, but not necessarily smooth nor convex. We assume also for much of the discussion that $c$ is separable, that is,

$$
c(\mathbf{x})=\sum_{i=1}^{n} c_{i}\left(x_{i}\right) .
$$

We also consider group (or block) separability, characterized by

$$
c(\mathbf{x})=\sum_{i=1}^{m} c_{i}\left(\mathbf{x}_{[i]}\right),
$$

where $\mathbf{x}_{[1]}, \mathbf{x}_{[2]}, \ldots, \mathbf{x}_{[m]}$ are $m$ disjoint sub-vectors of $\mathbf{x}$. We are especially interested in cases in which $\nabla f(\mathbf{x})$ is inexpensive to compute, relative to the cost of computing/storing the Hessian of $f$.

This paper presents an approach to solving problems of the form (2) that has two desirable properties: a) it is computationally competitive with the state-of-the-art algorithms designed for the standard $\ell_{2}-\ell_{1}$ problem (1); b) it is versatile enough to handle a broad class of generalizations of (1), such as problems in which the $\ell_{1}$ regularizer is replaced with an $\ell_{p}$-norm or with a group-separable regularizer.

\subsection{Proposed Approach}

Our approach generates a sequence of iterates $\mathbf{x}^{k}, k=1,2, \ldots$ by solving separable subproblems of the following form:

$$
\mathbf{x}^{k+1} \in \arg \min _{\mathbf{z}}\left(\mathbf{z}-\mathbf{x}^{k}\right)^{T} \nabla f\left(\mathbf{x}^{k}\right)+\frac{\alpha_{k}}{2}\left\|\mathbf{z}-\mathbf{x}^{k}\right\|_{2}^{2}+\tau c(\mathbf{z}),
$$

where $\alpha_{k} \in \mathbb{R}^{+}$. We refer to this approach as SpaRSA (for Sparse Reconstruction by Separable Approximation).

Different variants of the approach are distinguished by different choices of $\alpha_{k}$. We focus on variants based on the formula proposed by Barzilai and Borwein (BB) [1] in the context of smooth nonlinear minimization; see also $[8,16]$. BB methods have also been applied to constrained problems [2], especially bound-constrained quadratic programs $[7,15,22]$. To our knowledge, BB methods have not been previously used for problems involving nonsmooth terms, though this usage is a natural extension of the basic idea. We also consider monotone variants, in which $\alpha_{k}$ is increased as necessary to force a decrease in the objective function at every step.

\subsection{Related Work}

SpaRSA is closely related to iterative shrinkage/thresholding (IST) (a.k.a. iterative denoising, thresholded Landweber, forwardbackward splitting) algorithms [6, 9, 11, 13, 14, 17]. The form of the subproblem is the same, but IST methods use a more conservative 
choice of $\alpha_{k}$. In fact, it can be argued that SpaRSA is a speeded-up IST with better performance resulting from variation of $\alpha_{k}$.

SpaRSA is also related to the GPSR (gradient projection for sparse reconstruction) method recently presented by the authors of this manuscript [15]. While matching the speed of GPSR on the $\ell_{2}-\ell_{1}$ case, SpaRSA can be generalized beyond that case.

\section{THE PROPOSED APPROACH}

\subsection{The SpaRSA Framework}

The SpaRSA framework for problem (2) is as follows.

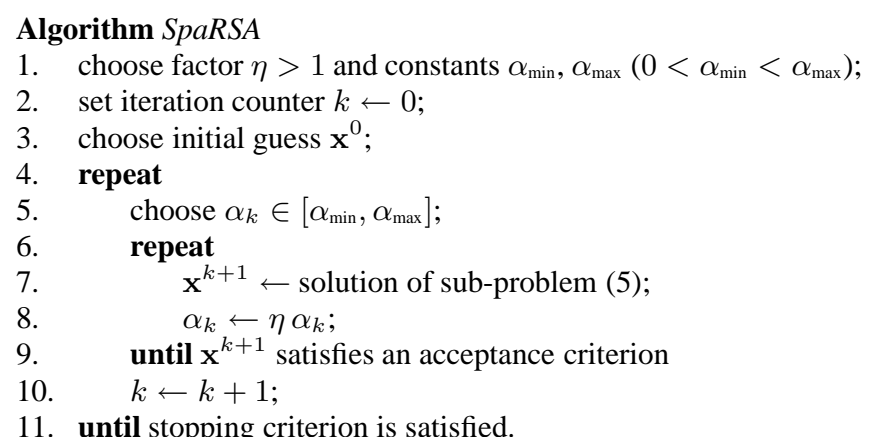

The several variants of SpaRSA are defined by two key steps of the algorithm: the choice of $\alpha_{k}$ (line 5) and the acceptance criterion (line 9). It is worth noting here that IST algorithms belong to the SpaRSA class. If $c$ is convex, if the acceptance criterion accepts any $\mathbf{x}^{k+1}$, and if we use a constant $\alpha_{k}$ satisfying the conditions given, e.g., in [6], we have a convergent IST algorithm. SpaRSA allows less conservative choices of $\alpha_{k}$, often leading to faster convergence.

\subsection{Solving the Subproblems}

By dropping irrelevant additive terms independent of $\mathbf{z}$, the subproblem (5) at line 7 of the algorithm can be rewritten as

$$
\mathbf{x}^{k+1} \in \arg \min _{\mathbf{z}} \frac{1}{2}\left\|\mathbf{z}-\mathbf{u}^{k}\right\|_{2}^{2}+\frac{\tau}{\alpha_{k}} c(\mathbf{z}),
$$

where $\mathbf{u}^{k}=\mathbf{x}^{k}-\nabla f\left(\mathbf{x}^{k}\right) / \alpha_{k}$. Since the term $\left\|\mathbf{z}-\mathbf{u}^{k}\right\|_{2}^{2}$ is a strictly convex function of $\mathbf{z}$, (6) has a unique solution when $c$ is convex. (For nonconvex $c$, there may exist several local minimizers.) In signal processing terms, (6) is called a denoising problem [13].

If $c$ has the separable form (3), the subproblem (6) is also separable and can be written as

$$
x_{i}^{k+1} \in \arg \min _{z} \frac{\left(z-u_{i}^{k}\right)^{2}}{2}+\frac{\tau}{\alpha_{k}} c_{i}(z), \quad i=1,2, \ldots, n .
$$

Separability is key to the efficiency of SpaRSA and IST algorithms. For some choices of $c_{i}$, the minimization in (7) has a unique closed form solution. When $c(\mathbf{z})=\|\mathbf{z}\|_{1}$ (thus $c_{i}(z)=|z|$ ), we have

$$
\arg \min _{z} \frac{\left(z-u_{i}^{k}\right)^{2}}{2}+\frac{\tau|z|}{\alpha_{k}}=\operatorname{soft}\left(u_{i}^{k}, \frac{\tau}{\alpha_{k}}\right),
$$

where $\operatorname{soft}(u, a) \equiv \operatorname{sign}(u) \max \{|u|-a, 0\}$ is the well-known softthreshold function.

Another notable case is the so-called $\ell_{0}$ quasi-norm $c(\mathbf{z})=$ $\|\mathbf{z}\|_{0}=\sum_{i} 1_{x_{i} \neq 0}$. In this case, we have

$$
\arg \min _{z} \frac{\left(z-u_{i}^{k}\right)^{2}}{2}+\frac{\tau}{\alpha_{k}} 1_{x_{i} \neq 0}=\operatorname{hard}\left(u_{i}^{k}, \sqrt{\frac{2 \tau}{\alpha_{k}}}\right),
$$

where $\operatorname{hard}(u, a) \equiv u 1_{|u|>a}$ is the hard-threshold function.

When $c_{i}(z)=|z|^{p}$, i.e., $c(\mathbf{z})=\|\mathbf{z}\|_{p}^{p}$, the closed form solution of (7) is known for $p=1$ (see (8)), $p=4 / 3, p=3 / 2$, and $p=2$. See $[5,6]$, for further details and theory about problems (6) and (7).

\subsection{Choosing $\alpha_{k}$ : The Barzilai-Borwein Method.}

In the most basic variant of the Barzilai-Borwein (BB) approach, we choose $\alpha_{k}$ such that $\alpha_{k} \mathbf{I}$ mimics the true Hessian $\nabla^{2} f(\mathbf{x})$ over the most recent step. Defining

$$
\mathbf{s}^{k}=\mathbf{x}^{k}-\mathbf{x}^{k-1}, \text { and } \mathbf{r}^{k}=\nabla f\left(\mathbf{x}^{k}\right)-\nabla f\left(\mathbf{x}^{k-1}\right),
$$

we require that $\alpha_{k} \mathbf{s}^{k} \approx \mathbf{r}^{k}$ in the least-squares sense, leading to

$$
\alpha_{k}=\arg \min _{\alpha}\left\|\alpha \mathbf{s}^{k}-\mathbf{r}^{k}\right\|_{2}^{2}=\left(\mathbf{s}^{k}\right)^{T} \mathbf{r}^{k} /\left[\left(\mathbf{s}^{k}\right)^{T} \mathbf{s}^{k}\right] .
$$

When $f(\mathbf{x})=(1 / 2)\|\mathbf{A} \mathbf{x}-\mathbf{y}\|_{2}^{2}$, the previous expression becomes $\alpha_{k}=\left\|\mathbf{A} \mathbf{s}^{k}\right\|_{2}^{2} /\left\|\mathbf{s}^{k}\right\|_{2}^{2}$. These formulas can be safeguarded appropriately to ensure that $\alpha_{k}$ remains in the range $\left[\alpha_{\min }, \alpha_{\max }\right]$.

\subsection{The Acceptance Criterion}

In the simplest variant of the SpaRSA scheme, the acceptance criterion is trivial: accept whatever $\mathbf{z}$ solves the subproblem (5) as the new iterate $\mathbf{x}^{k+1}$, even if it yields an increase in the objective function $\phi$. We consider also a variant in which $\alpha_{k}$ is viewed as a damping parameter in the subproblem (6), which is increased until the solution of this subproblem yields a decrease in $\phi$. In this scheme, the acceptance criterion may be $\phi\left(\mathbf{x}^{k+1}\right)<\phi\left(\mathbf{x}^{k}\right)$, or we may enforce a more stringent variant that requires the margin of decrease to be at least some (positive constant) multiple of the decrease promised by the subproblem (5). The initial choice of $\alpha_{k}$ can be given by (10), or by modifying the value $\alpha_{k-1}$ from the previous iteration. We call the former variant of the algorithm SpaRSA-monotone.

The existence of a value of $\alpha_{k}$ sufficiently large to ensure a decrease in the objective at each iteration can be inferred from the connection between (6) and the following trust-region subproblem:

$$
\min _{\mathbf{z}} \nabla f\left(\mathbf{x}^{k}\right)^{T}\left(\mathbf{z}-\mathbf{x}^{k}\right)+\tau c(\mathbf{z}) \text { subject to }\left\|\mathbf{z}-\mathbf{x}^{k}\right\|_{2} \leq \Delta_{k} .
$$

It also follows from the known fact, which underlies the monotonicity of IST algorithms [14], that there is a constant $\bar{\alpha}>0$ such that descent is assured whenever $\alpha_{k} \geq \bar{\alpha}$.

\subsection{Warm Starting and Continuation}

The SpaRSA approach benefits from a good starting point $\mathbf{x}^{0}$, which suggests that we can use the solution of (2), for a given value of $\tau$, to initialize SpaRSA in solving (2) for a nearby value of $\tau$. The second run will typically be significantly faster than the first one. An important application of warm-starting is continuation, as recently suggested in [17]. The speed of SpaRSA algorithms may degrade considerably for smaller values of the regularization parameter $\tau$. However, if we use SpaRSA to solve (2) for a larger value of $\tau$, then decrease $\tau$ in steps toward its desired value, running SpaRSA with warm-start for each successive value of $\tau$, we are often able to identify the solution much more efficiently than if we just ran SpaRSA once for the desired value of $\tau$ from a "cold start." 


\section{GROUP-SEPARABLE REGULARIZERS}

In this section we consider group-separable (GS) regularizers of the form (4). In this case, the minimization (6), instead of decoupling into a set of one-dimensional minimizations (7), decouples into a set of $m$ independent multi-dimensional minimizations, of the form

$$
\min _{\mathbf{w} \in \mathbb{R}^{l}} \frac{1}{2}\|\mathbf{w}-\mathbf{b}\|_{2}^{2}+\beta \Phi(\mathbf{w}),
$$

where $l$ is the dimension of $\mathbf{x}_{[i]}, \mathbf{b}=\mathbf{u}_{[i]}^{k}, \Phi=c_{i}$, and $\beta=\tau / \alpha_{k}$.

GS regularizers are desirable when there exists a group structure in $\mathbf{x}$, which arises naturally in many applications.

- In brain imaging, the voxels associated with different functional regions (e.g., motor or visual cortices) may be grouped together in order to identify a sparse set of regional events. In $[3,4]$, an EM algorithm (equivalent to IST) was proposed for solving problems of this type.

- A GS- $\ell_{2}$ penalty $\left(\Phi(\mathbf{w})=c_{i}(\mathbf{w})=\|\mathbf{w}\|_{2}\right)$ was proposed for source localization in sensor arrays [20]; second-order cone programming was used to solve the optimization problem.

- In gene expression analysis, some genes are organized in functional groups. This has motivated an approach called CAP (composite absolute penalty) [25], which has the form (4), and uses a greedy optimization scheme [26].

GS regularizers have also been proposed for ANOVA regression models [19, 21, 24], and Newton-type optimization methods have been proposed in that context. An interior-point method for the GS$\ell_{\infty}$ case $\left(\Phi(\mathbf{w})=c_{i}(\mathbf{w})=\|\mathbf{w}\|_{\infty}\right)$ was proposed in [23]. The SpaRSA framework is versatile enough to handle the GS regularizes arising all in the applications described above.

As in $[5,6]$, convex analysis can be used to obtain the solution of (11). If $\Phi$ is a norm, it is proper, convex (maybe not strictly so), and homogenous. Since the quadratic term in (11) is proper and strictly convex, this problem has a unique solution, which can be written explicitly as follows:

$$
w=\mathbf{b}-P_{\beta C_{\Phi}}(\mathbf{b}),
$$

where $P_{B}$ denotes the orthogonal projector onto set $B$, and $C_{\Phi}$ is a 1-ball in the dual norm $\Phi^{\star}$, that is, $C_{\Phi}=\left\{\mathbf{w} \in \mathbb{R}^{l}: \Phi^{\star}(\mathbf{w}) \leq 1\right\}$.

For $\Phi(\mathbf{w})=\|\mathbf{w}\|_{2}$, the dual norm is also $\Phi^{\star}(\mathbf{w})=\|\mathbf{w}\|_{2}$, thus $\beta C_{\|\cdot\|_{2}}=\left\{\mathbf{w} \in \mathbb{R}^{l}:\|\mathbf{w}\|_{2} \leq \beta\right\}$. Clearly, if $\|\mathbf{b}\|_{2} \leq \beta$, then $P_{\beta C_{\|\cdot\|_{2}}}(\mathbf{b})=\mathbf{b}$, thus $\mathbf{b}-P_{\beta C_{\|\cdot\|_{2}}}(\mathbf{b})=0$. If $\|\mathbf{b}\|_{2}>\beta$, then $P_{\beta C_{\|\cdot\|_{2}}}(\mathbf{b})=\beta \mathbf{b} /\|\mathbf{b}\|_{2}$. These two cases are written compactly as

$$
w=\frac{\mathbf{b}}{\|\mathbf{b}\|_{2}} \max \left\{\|\mathbf{b}\|_{2}-\beta, 0\right\} .
$$

Naturally, if $l=1$, (13) reduces to the scalar soft-threshold (8).

For $\Phi(\mathbf{w})=\|\mathbf{w}\|_{\infty}$, the dual norm is $\Phi^{\star}(\mathbf{w})=\|\mathbf{w}\|_{1}$, thus $\beta C_{\|\cdot\|_{\infty}}=\left\{\mathbf{w} \in \mathbb{R}^{n}:\|\mathbf{w}\|_{1} \leq \beta\right\}$. In this case, the solution of (11) is the residual of the orthogonal projection of $\mathbf{b}$ onto the $\ell_{1} \beta$ ball. This projection (thus also the residual) can be computed with $O(l \log l)$ cost, as recently shown in $[3,4,10]$.

\section{EXPERIMENTS}

\subsection{Speed Comparisons for the $\ell_{2}-\ell_{1}$ Problem}

The purpose of our first experiment is to compare SpaRSA with the state-of-the-art algorithms IST and GPSR (see Subsection 1.3), and the $l 1 \_l s$ method [18], in a typical CS scenario (as in $[15,18]$ ): $f(\mathbf{x})=\|\mathbf{A} \mathbf{x}-\mathbf{y}\|_{2}^{2}$, with $\mathbf{A}$ a $2^{10} \times 2^{12}$ random matrix; $\mathbf{y}$ is generated as $\mathbf{y}=\mathbf{A} \mathbf{x}_{\text {true }}+\mathbf{e}$, where $\mathbf{e}$ is a Gaussian white vector with variance $10^{-4}$, and $\mathbf{x}_{\text {true }}$ is a vector with 160 randomly placed \pm 1 spikes and zeros elsewhere. We use the $\ell_{1}$ regularizer $c(\mathbf{x})=\|\mathbf{x}\|_{1}$, and $\tau=0.1\left\|\mathbf{A}^{T} \mathbf{y}\right\|_{\infty}$, as in $[15,18]$. In this (and all other) experiments, $\alpha_{\max }=1 / \alpha_{\min }=10^{30}$ and $\eta=2$ (for SpaRSA-monotone). To perform the comparison, independently of the adopted stopping rule, we first run $l 1 \_l s$ and then the other algorithms until each reaches the same value of the objective function reached by $l 1 \_l s$. Table 1 reports the CPU times required by SpaRSA, two variants of GPSR, $l 1 \_l s$, and IST, as well as the final mean squared error (MSE) of the

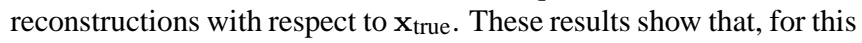
$\ell_{2}-\ell_{1}$ problem, SpaRSA is slightly faster than GPSR and clearly faster than $l 1 \_l s$ and IST, while achieving a similar value of MSE.

Table 1. CPU times (average over 10 runs) of several algorithms on the CS experiment described in the text.

\begin{tabular}{|l|c|l|}
\hline Algorithm & CPU time (secs.) & MSE \\
\hline \hline SpaRSA & 0.44 & $2.42 \mathrm{e}-3$ \\
SpaRSA-monotone & 0.45 & $2.49 \mathrm{e}-3$ \\
GPSR-BB & 0.55 & $2.81 \mathrm{e}-3$ \\
GPSR-Basic & 0.69 & $2.59 \mathrm{e}-3$ \\
$l 1 \_l s$ & 6.56 & $2.51 \mathrm{e}-3$ \\
IST & 2.76 & $2.51 \mathrm{e}-3$ \\
\hline
\end{tabular}

An indirect comparison with other codes can be made via [18, Table 1], which shows that $l 1 \_l s$ outperforms the method from [12] (6.9 vs 11.3 secs.), as well as $\ell_{1}$-magic by about two orders of magnitude and pdco from SparseLab by about one order of magnitude.

The second experiment assesses how the computational cost of SpaRSA grows with the size of matrix $\mathbf{A}$, using a setup similar to the one in $[15,18]$. Assuming that the computational cost is $O\left(n^{\gamma}\right)$, we obtain empirical estimates of $\gamma$. SpaRSA and SpaRSA-monotone have empirical exponents of .88 and .87 , respectively, similar to the values .86 and .87 of GPSR and GPSR-Basic. IST has a similar exponent .89 , but a worse constant. For $l 1 \_l s$, we found $\gamma=1.21$, in agreement with the value 1.2 reported in [18].

\subsection{Group-Separable Regularizers}

Here we illustrate the use of SpaRSA with the GS regularizers considered in Section 3. In our example, $x_{\text {true }}$ is a $2^{12}$-dimensional vector, divided into $m=64$ groups of length $l_{i}=64$. As above, $\mathbf{A}$ a $2^{10} \times 2^{12}$ random matrix and $\mathbf{y}$ is generated as $\mathbf{y}=\mathbf{A} \mathbf{x}_{\text {true }}+\mathbf{e}$, where $\mathbf{e}$ is Gaussian white noise with variance $10^{-4}$. To generate $\mathbf{x}_{\text {true }}$, we randomly choose 8 groups and fill them with zero-mean Gaussian random samples of unit variance; all other groups are filled with zeros. Finally we run SpaRSA, with $f(\mathbf{x})=\|\mathbf{A x}-\mathbf{y}\|_{2}^{2}$ and $c(\mathbf{x})$ as given by (4), where $c_{i}\left(\mathbf{x}_{[i]}\right)=\left\|\mathbf{x}_{[i]}\right\|_{2}$. The value of $\tau$ is hand-tuned for optimal performance. Fig. 1 shows the result obtained by SpaRSA, based on the GS- $\ell_{2}$ regularizer, which successfully recoverers the group structure of $\mathbf{x}_{\text {true }}$, as well as the result obtained with the classical $\ell_{1}$ regularizer, for the best choice of $\tau$.

In the second experiment, we consider a similar scenario, with a single difference. Each active group, instead of being filled with Gaussian random samples, is filled with ones. This case is clearly more adequate for a GS- $\ell_{\infty}$ regularizer, as illustrated in Fig. 2 , which achieves an almost perfect reconstruction, with an MSE 2 orders of magnitude smaller than what is obtained with a GS- $\ell_{2}$ regularizer. 

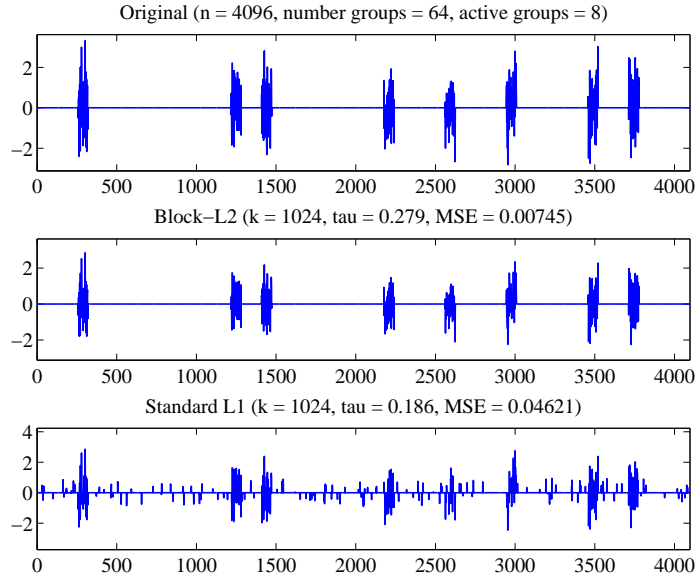

Fig. 1. Comparison of GS- $\ell_{2}$ regularizer with conventional $\ell_{1}$ regularizer. Exploiting known group structure provides a dramatic gain.
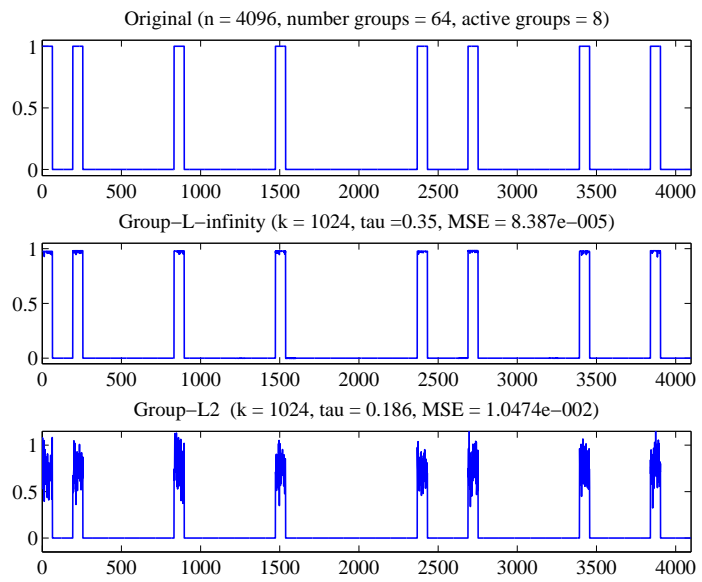

Fig. 2. Comparison of GS- $\ell_{2}$ and GS- $\ell_{\infty}$ regularizers. Signals with uniform behavior within groups benefit from the GS- $\ell_{\infty}$ regularizer.

\section{CONCLUDING REMARKS}

In this paper, we have introduced the SpaRSA algorithmic framework for solving large-scale optimization problems involving the sum of a smooth error term and a possibly nonsmooth regularizer. We give experimental evidence that SpaRSA matches the speed of the state-of-the-art method when applied to the $\ell_{2}-\ell_{1}$ problem, and show that SpaRSA can be generalized to other regularizers such as those with group-separable structure. Ongoing work includes a more thorough experimental evaluation involving wider classes of regularizers, and theoretical analysis of the convergence properties.

\section{REFERENCES}

[1] J. Barzilai, J. Borwein, "Two point step size gradient methods," IMA Journal of Numerical Analysis, vol. 8, pp. 141-148, 1988.

[2] E. Birgin, J. Martinez, M. Raydan, "Nonmonotone spectral projected gradient methods on convex sets," SIAM Journal on Optimization, vol. 10, pp. 1196-1211, 2000.

[3] A. Bolstad, B. Van Veen, R. Nowak, "Space-time sparsity regularization for the magnetoencephalography inverse problem", Proc. of the IEEE Itern. Conference on Biomedical Imaging, Arlington, VA, 2007.
[4] A. Bolstad, B. Van Veen, R. Nowak, R. Wakai, "An expectationmaximization algorithm for space-time sparsity regularization of the MEG inverse problem", Proc. of the Itern. Conference on Biomagnetism, Vancouver, BC, Canada, 2006.

[5] C. Chaux, P. Combettes, J.-C. Pesquet, V. Wajs, "A variational formulation for frame-based inverse problems," Inverse Problems, vol. 23, pp. 1495-1518, 2007.

[6] P. Combettes, V. Wajs, "Signal recovery by proximal forwardbackward splitting," SIAM Journal on Multiscale Modeling \& Simulation, vol. 4, pp. 1168-1200, 2005.

[7] Y.-H. Dai, R. Fletcher. "Projected Barzilai-Borwein methods for largescale box-constrained quadratic programming," Numerische Mathematik, vol. 100, pp. 21-47, 2005.

[8] Y.-H. Dai, W. Hager, K. Schittkowski, H. Zhang, "The cyclic BarzilaiBorwein method for unconstrained optimization," IMA Journal of $\mathrm{Nu}$ merical Optimization, vol. 26, pp. 604-627, 2006.

[9] I. Daubechies, M. Defriese, C. De Mol, "An iterative thresholding algorithm for linear inverse problems with a sparsity constraint", Comm. on Pure and Applied Math., vol. LVII, pp. 1413-1457, 2004.

[10] I. Daubechies, M. Fornasier, I. Loris, "Accelerated projected gradient method for linear inverse problems with sparsity constraints." Preprint available at www.dsp.ece.rice.edu/cs, 2007.

[11] M. Elad, "Why simple shrinkage is still relevant for redundant representations?", IEEE Trans. Inf. Th., vol. 52, pp. 5559-5569, 2006.

[12] D. Donoho and Y. Tsaig. "Fast solution of L1-norm minimization problems when the solution may be sparse," Technical Report, Institute for Computational and Math. Eng., Stanford University, 2006.

[13] M. Figueiredo, R. Nowak, "An EM algorithm for wavelet-based image restoration," IEEE Trans. Image Proc., vol. 12, pp. 906-916, 2003.

[14] M. Figueiredo, R. Nowak, "A bound optimization approach to waveletbased image deconvolution," IEEE Intern. Conf. on Image Processing - ICIP'05, Genoa, Italy, 2005.

[15] M. Figueiredo, R. Nowak, S. Wright, "Gradient projection for sparse reconstruction: application to compressed sensing and other inverse problems", IEEE Journal of Selected Topics in Signal Processing, 2007 (to appear). Available at www.lx.it.pt/ $\mathrm{mtf} / \mathrm{GPSR}$

[16] L. Grippo, M. Sciandrone, "Nonmonotone globalization techniques for the Barzilai-Borwein method," Computational Optimization and Applications, vol. 32, pp. 143-169, 2002.

[17] T. Hale, W. Yin, Y. Zhang, "A fixed-point continuation method for $\ell_{1}$-regularized minimization with applications to compressed sensing." TR07-07, Dept. of Comput. and Appl. Math., Rice University, 2007.

[18] S. Kim, K. Koh, M. Lustig, S. Boyd, and D. Gorinvesky. "A method for large-scale $\ell_{1}$-regularized least squares problems with applications in signal processing and statistics," Tech. Report, Dept. of Electrical Engineering, Stanford University, 2007.

[19] Y. Kim, J. Kim, Y. Kim, "Blockwise sparse regression", Statistica Sinica, vol. 16, 2006.

[20] D. Malioutov, M. Cetin, A. Willsky, "Sparse signal reconstruction perspective for source localization with sensor arrays", IEEE Trans. Signal Processing, vol. 53, pp. 3010-3022, 2005.

[21] L. Meier, S. van de Geer, P. Buhlmann, "The group LASSO for logistic regression", Research Report 131, ETH-Zurich, 2006.

[22] T. Serafini, G. Zanghirati, L. Zanni. "Gradient projection methods for large quadratic programs and applications in training support vector machines," Optim. Meth. and Software, vol. 20, pp. 353-378, 2004.

[23] B. Turlach, W. N. Venables, and S. J. Wright. "Simultaneous variable selection", Technometrics, vol. 27, pp. 349-363, 2005.

[24] M. Yuan, Y. Lin, "Model selection and estimation in regression with grouped variables", J. Royal Stat. Soc. B, vol. 68, pp. 49-67, 2006.

[25] P. Zhao, G. Rocha, B. Yu, "Grouped and hierarchical model selection through composite absolute penalties", TR 703, Statistics Department, University of California - Berkeley, 2007.

[26] P. Zhao, B. Yu, "Boosted Lasso", Technical Report, Statistics Department, University of California - Berkeley, 2004. 